\title{
ADHD Deficit as Measured in Adolescent Boys with a Continuous Performance Task Is Related to Antenatal Maternal Anxiety
}

\author{
BEA R.H. VAN DEN BERGH, MAARTEN MENNES, VEERLE STEVENS, JAAP VAN DER MEERE, NORBERT BÖRGER, \\ PETER STIERS, ALFONS MARCOEN, AND LIEVEN LAGAE
}

\begin{abstract}
Department of Psychology [B.R.H.V., M.M., A.M.], Catholic University of Leuven, 3000 Leuven, Belgium; Department of Pediatric Neurology [M.M., P.S., L.L.], Catholic University of Leuven, University Hospital Gasthuisberg, 3000 Leuven, Belgium; Department of Communication Disorders [V.S.], University Hospital Antwerp, 2650 Edegem, Belgium; Laboratory of Developmental and Experimental Clinical Psychology [J.V., N.B.], University of Groningen, 9712 TS Groningen, The Netherlands
\end{abstract}

\begin{abstract}
Antenatal maternal anxiety has been shown to be related to infant temperament, childhood disorders, and impulsivity in adolescence. This study prospectively investigated whether antenatal maternal anxiety is associated with performance on a continuous performance task. Sixty-four adolescents (mean age, 15 y; 34 boys, 30 girls) were examined with a computerized continuous performance task (CPT) measuring sustained attention. Results showed that the CPT performance of boys of mothers with high levels of state anxiety during the 12 th to 22 nd postmenstrual week of pregnancy declined as the task progressed: their processing speed became slower and the variability in their reaction times increased. The study controlled for the possible confounding influences of postnatal maternal anxiety, the parents' educational level, and intelligence. Establishing a link between antenatal maternal anxiety and an objective measure of sustained attention/self-regulation, our results extend the growing evidence for an association between antenatal maternal anxiety and the neurobehavioral development of the offspring up into adolescence. (Pediatr Res 59: 78-82, 2006)
\end{abstract}

A growing number of studies provide evidence that child development is negatively related to heightened maternal anxiety and stress during pregnancy (1-3). Prospective longitudinal studies, using behavioral questionnaires and clinical scales administered at several moments during development up to $9 \mathrm{y}$ of age, revealed that children of mothers with high anxiety during pregnancy had more difficulties with cognitive, behavioral, and emotional self-regulation as expressed in infant difficult temperament, attention regulation problems, hyperactivity, clinical diagnosis of ADHD, ADHD symptoms, conduct disorders, and emotional problems (4-9). One explanation for these observations, which is based on experimental studies in rodents and other animals, is that, during sensitive periods of development, disturbing factors

Received March 21, 2005; accepted June 27, 2005

Correspondence: Bea R.H. Van den Bergh, Ph.D., Department of Psychology, Catholic University of Leuven, Tiensestraat 102, B-3000 Leuven, Belgium; e-mail: Bea. vandenbergh@psy.kuleuven.be

This research is supported by Grant number G.0211.03 of the Fund for Scientific Research (Flanders, Belgium) and by the Population and Family Study Center, Brussels, Belgium. L.L. is holder of the UCB Chair on Cognitive Dysfunctions in Childhood the Catholic University of Leuven.

DOI: 10.1203/01.pdr.0000191143.75673.52 (hormones related to maternal anxiety and/or stress) exercise organizational effects that constrain the malleability of biologic systems that ultimately can predispose to disorder $(10,11)$.

At present, it is not clear during which prenatal period the human fetus is most sensitive to the negative influence of the mother's emotional state. O'Connor et al. $(7,8)$ found anxiety at $32 \mathrm{wk}$ of pregnancy to be related to behavioral and emotional problems of both boys and girls, whereas anxiety at 18 wk of pregnancy was only in girls associated with these problems. In the second wave of their longitudinal study, Van den Bergh and Marcoen (9) pinpointed 12-22 wk as the only important period predictive of ADHD symptoms and externalizing and internalizing problems. In the first part of the third wave of this study, maternal anxiety during this period was found to be related to impulsivity during performance on a divided attention task and to lower scores on two intelligence subtests (12).

This article represents a second part of the third wave of the prospective study of Van den Bergh and Marcoen (9) and is aimed at investigating whether a relation can be established between maternal anxiety during pregnancy and a specific aspect of self-regulation (sustained attention) in 14/15-y-old adolescents.

In the CPT used, sustained attention is understood as the ability to self-sustain mindful conscious processing of stimuli whose repetitive, nonarousing qualities would otherwise lead to habituation and distraction to other stimuli (13). Successful performance on a CPT requires that one is able to endogenously (i.e. autonomously, from within oneself and without external sources) modulate alertness by resisting to potentially distracting stimuli $(13,14)$. It is thought that both structurally

Abbreviations: ACC, anterior cingulate cortex; ADHD, attention-deficit hyperactivity disorder; CPT, continuous performance task; PFC, prefrontal cortex 
and functionally the organization of the medial PFC (including the ACC) is ideally to participate in this kind of "willed control" of behavior (15) or in "effortful" (16), "volitional (not necessary conscious) control" (17) concepts closely related to self-regulation.

\section{METHODS}

Participants. Eighty-six healthy mothers and their firstborn children started the longitudinal study $(9,12)$. Sixty-four mother-child pairs participated in the current part of the third wave. This group comprised 33 boys and 31 girls, mean age was 15 y (range, $14.54-15.54 \mathrm{y} ; \mathrm{SD}=3 \mathrm{mo}$ ). All babies were born in hospital between 36 and 41 wk of gestation. Their mean birth weight was $3326.56 \mathrm{~g}(\mathrm{SD}=482.15 \mathrm{~g})$.

The ethical committee for experiments on human beings of the University Hospital Gashuisberg, Leuven, approved the study. All participants gave their informed consent.

Antenatal maternal anxiety. At 12-22, 23-31, and 32-40 wk of pregnancy, anxiety of the mother was measured using the Dutch version of the State Trait Anxiety Inventory (STAI), a self-report questionnaire $(18,19)$. Trait anxiety refers to a disposition or proneness to react with anxiety, whereas state anxiety refers to a transient emotional state, characterized by subjectively experienced tension and an increased activity state of the autonomous nervous system. Because the state anxiety subscale provides a valid measure of the intensity of transitory anxiety in response to real-life stress (20), state (and not trait) anxiety was used in the present investigation of the effects of antenatal maternal anxiety.

All analyses were carried out with two anxiety groups: a low-to-averageanxiety group (state anxiety score $<75$ th percentile) and a high-anxiety group (state anxiety score $\geq 75$ th percentile). Percentiles were calculated for each successive period of pregnancy during which the questionnaire was administered.

Sustained attention/self-regulation. Because of its extremely slow presentation rate, we used the CPT O/Q task that was used in the study by Van der Meere et al. (21). Moreover, this task is presented with a variable interstimulus interval so participants cannot anticipate for the next trial. This is important inasmuch as the ability to anticipate on forthcoming trials may camouflage attentional deficits (22). In the task, the letter $\mathrm{O}$ was presented in the middle of a computer screen. During the test, which lasted 24 min, the O changed 57 times into a $\mathrm{Q}$ and back to the letter O. Participants were instructed to press a button (spacebar) as fast as possible as soon as the $\mathrm{O}$ changed into a $\mathrm{Q}$. The presentation time of the target letter $\mathrm{Q}$ was either 8, 4, or $2 \mathrm{~s}$. The time between two Qs ranged from 10 to $52 \mathrm{~s}$, with an average of $24 \mathrm{~s}$. Each participant received a 2-min training session before the experiment started. The CPT was introduced after the training and participants were asked to complete the test without interruption. During the test, the experimenter was seated outside the visual field of the participant. Talking during the experiment was not permitted and was ignored if initiated by the participant.

The mean reaction time (measure of processing speed), the SD of the reaction time (SD; measure of consistency in responding and ability to sustain attention), the number of omissions (no response when target letter Q appeared; measure of inattention), and the number of commissions (response when $\mathrm{O}$ was present; measure of hyperactivity/impulsivity) were used as dependent measures in our analyses. In our design, time-on-task was operationalized by comparing the first 4 min of the task execution (block 1) with the last 4 min (block 6). This is because sustained attention problems are defined as a performance decline over time. However, this decline is generally not a linear function because subjects protect their performance over time by mobilizing extra effort. Therefore, comparing the first with the last block of task performance provides an index of the maximal decline over time.

Data analysis. The relationship between maternal state anxiety at each of the three pregnancy periods and the CPT measures was examined with analysis of covariance (MANCOVA and subsequent ANCOVA). A significance level of 0.05 was used.

Potential covariates. Because the three anxiety periods were intercorrelated (see Table 1), we included the maternal state anxiety measures of the two other pregnancy periods as covariates in the analysis for each maternal state anxiety measure. Other included covariates were postnatal trait anxiety, which correlated with all three prenatal state anxiety measures, the parents' educational level, and age of the adolescents, which were significantly correlated with different measures of task performance in block 6 of the CPT (for the parents' educational level: mean reaction time: $r=-0.27, p=0.03$; SD of the reaction time: $r=-0.25, p=0.04$; commission errors: $r=$ $-0.0127, p=\mathrm{NS}$; omission errors: $r=-0.26, p=0.04$; for age: mean reaction time: $r=0.36, p=0.003$; $\mathrm{SD}$ of the reaction time: $r=0.30, p=$
Table 1. Descriptive statistics of state anxiety and correlations with potential covariates

\begin{tabular}{lccc}
\hline & \multicolumn{3}{c}{ Prenatal state anxiety } \\
\cline { 2 - 4 } Descriptive statistics & State $12-22$ & State $23-31$ & State $32-40$ \\
\hline Total group & $n=64$ & $n=64$ & $n=64$ \\
Mean & 39.3 & 34.7 & 36.1 \\
SD & 8.7 & 8.6 & 7.3 \\
Range & $24-62$ & $20-62$ & $20-58$ \\
75th Percentile & 43.5 & 39.2 & 40.1 \\
Low-to-average-anxiety group & $n=48$ & $n=48$ & $n=48$ \\
Mean & 35.2 & 30.9 & 33.2 \\
SD & 4.7 & 5.5 & 5.2 \\
Range & $24-43$ & $20-39$ & $20-40$ \\
High-anxiety group & $n=16$ & $n=16$ & $n=16$ \\
Mean & 51.5 & 46.0 & 45.0 \\
SD & 5.9 & 6.2 & 5.3 \\
Range & $44-62$ & $40-62$ & $41-58$ \\
Correlations & & & \\
State anxiety 12-22 wk & - & $0.56^{* *}$ & 0.19 \\
State anxiety 23-31 wk & & - & $0.52^{* *}$ \\
Postnatal trait anxiety & $0.38^{* *}$ & $0.66^{* *}$ & $0.63^{* *}$ \\
\hline
\end{tabular}

$\S$ Phi-coefficient on dichotomous variables. $* p<0.05$. ** $p<0.01$.

0.014; commission errors: $r=0.27, p=0.028$; omission errors: $r=-0.03$, $p=\mathrm{NS}$ ). Including other covariates was not possible because of the small sample size. In addition, out of 22 correlations between smoking during pregnancy, birth weight and the predictor and outcome variables only one reached significance; smoking correlated with the number of omission errors in block $6(r=0.25, p=0.047)$.

Finally, to verify whether results on the CPT could not be reduced to differences in intellectual abilities between adolescents of high versus lowaverage anxious pregnant mothers, the standard scores of both vocabulary and block design [Weschler Intelligence Scale for Children (WISC-R)] $(23,24)$ were entered as covariates in all analyses. This procedure is justified by the fact that adolescents of highly anxious pregnant women indeed showed lower cognitive abilities as measured with these two tasks (12).

\section{RESULTS}

Descriptives for prenatal state anxiety measures. The mean state anxiety scores for the total group differed depending on the pregnancy period (see Table 1 for descriptive statistics). State 12-22 was higher than state 23-31 ( $t=3.0, p$ $<0.003)$ and State 32-40 $(t=2.2 ; p=0.027)$. State 23-31 and state $32-40$ did not differ $(t=-1.0 ; p=0.31)$. The anxiety scores in our research population are similar to those in the nonclinical female Dutch community sample described in the STAI manual (19). The 75th percentile calculated for each anxiety period approximately equals decile 7 (for state 1222) and decile 6 (for state 23-31 and state 32-40). Decile 7 includes scores ranging from 41 to 45 , decile 6 includes scores from 38 to 40 .

Sustained attention/self-regulation. The relationship between each prenatal maternal state anxiety measure and the dependent variables of the CPT was first analyzed with repeated measures (M)ANCOVA, with anxiety as betweensubjects variable and time-on-task (block 1 versus block 6) as within-subject variable. As indicated above, in (M)ANCOVA of each state anxiety measures the two other state anxiety measures as well as postnatal trait anxiety, the intellectual abilities of the adolescents, parental educational level and age of the children were entered as covariates. 
The results of these analyses did not yield any associations between antenatal maternal anxiety and the sustained attention task. Children of mothers with high state 12-22, state 23-31, or state 32-40 anxiety did not differ for any of the performance measures from children of mothers with low-toaverage anxiety (highest $F$ value for state $23-31, F(4,52)=$ $2.35, p=0.07)$.

However, as pointed out by Van den Bergh and Marcoen (9), it could be that boys of highly anxious mothers are more at risk for developing self-regulation problems and behavioral disorders than girls of highly anxious mothers. Therefore, the analyses were recalculated with gender as a second betweensubjects factor. The general MANCOVA for state 12-22 was significant $(F(4,50)=5.50, p<0.001)$. Individual ANCOVA then resulted in significant state $12-22 \times$ gender $\times$ time-ontask interactions for both mean reaction time $(F(1,53)=5.61$; $p=0.02)$ and the SD of the reaction time $(F(1,53)=13.12$; $p<0.001)$. As can be seen in Figure 1, the mean reaction time and the SD of the boys in the high-anxiety group increased as the task proceeded. This was confirmed statistically by planned comparisons analysis of the interaction between state 12-22 and time-on-task in both boys and girls separately. For the boys, these analyses resulted in a significant effect on mean reaction time $(F(1,53)=4.02 ; p=0.05)$ and a significant effect on $\mathrm{SD}$ of the reaction time $(F(1,53)=6.88 ; p=$ $0.01)$. For the girls, there was a significant effect for SD of the reaction time $(F(1,53)=4.84 ; p=0.03)$, which decreased, but not for the mean reaction time, remaining stable. No effects were found for the amount of commissions or omissions made.

The general state $23-31 \times$ gender $\times$ time-on-Task MANCOVA was also significant $(F(4,50)=5.52, p<0.001)$. Individual ANCOVA for each dependent variable again resulted in significant state $23-31 \times$ gender $\times$ time-on-task interaction for the mean reaction time $(F(1,53)=4.64 ; p=$ $0.03)$ and SD of the reaction time $(F(1,53)=19.95 ; p<$ 0.001 ). Planned comparisons revealed that in boys there was no interaction between state 23-31 and time-on-task for mean reaction time and $\mathrm{SD}$ of the reaction time, whereas in girls these interactions were significant $(F(1,53)=11.24 ; p<$
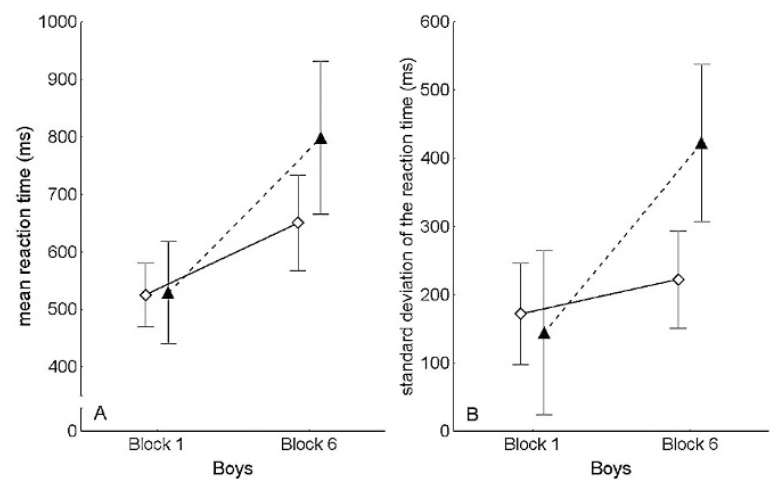

Figure 1. State $12-22 \times$ gender $\times$ time-on-task interaction for reaction time $(A ; p=0.02)$ and the SD of the reaction time $(B ; p<0.001)$ on the CPT. Only the results for the boys are shown. Black triangles: children of mothers who experienced high levels of anxiety during the 12th to 22nd week of pregnancy ( $n=11)$; open diamonds: children of mothers who experienced low-toaverage levels of anxiety $(n=23)$. Error bars depict $95 \%$ confidence interval.
0.001 for mean reaction time and $F(1,53)=28.20 ; p<0.001$ for $\mathrm{SD}$ of the reaction time). The effects for the mean reaction time and SD of the reaction time are probably due to a very high reaction time and SD of the five girls of the highly anxious group in block 1. The reason for these high initial values was not clear. In addition, they already returned to normal values in the second and third block (mean reaction time in block 1: 684.7; block 2: 581.1; block 3: 606.8; and SD of the reaction time in block 1: 541.8; block 2: 329.3; block 3: 217.9). No effects were found for the amount of commissions and omissions.

The general anxiety $\times$ gender $\times$ time-on-Task MANCOVA for state $31-40$ was not significant $(F(4,50)=$ $0.45 ; p=0.77)$.

\section{DISCUSSION}

This study investigated the hypothesis that adolescents of mothers who experienced high levels of anxiety at $12-22 \mathrm{wk}$ of pregnancy show more difficulties with sustained attention/ self-regulation than adolescents of pregnant mothers with low or moderate anxiety levels in that period. Our results confirmed this hypothesis for boys but not for girls. These results are particularly robust inasmuch as the study controlled for confounding factors such as intellectual abilities of the adolescents, maternal anxiety in the two other pregnancy periods, postnatal maternal anxiety, the parents' educational level, and age of the adolescents. The results of this study imply that maternal anxiety may change the developmental path of the child from before birth. Whether this path leads to pathology probably depends on the genetic liability of the child, on specific positive and negative characteristics of the postnatal environment, and interaction with caregivers (25).

Significant associations with antenatal maternal anxiety were found in the mean reaction time and SD of the reaction time but not in the number of errors they made. Although there was no difference in reaction time or variance of reaction time at the beginning of the task, boys of mothers who were highly anxious during the 12th to 22nd week of pregnancy responded significantly more variably near the end of this long and tedious task. In addition, they responded slower as the task proceeded. These results indicate that boys of highly anxious mothers were less able to sustain their attention and stay focused to the task at hand. The fact that these results were found after controlling for the scores on vocabulary and block design indicates that maternal anxiety is associated with sustained attention/self-regulation independent of the intellectual abilities measured in these two intelligence subtests.

One interpretation of the performance on the CPT holds that boys in the high-anxiety group were not optimally aroused (or motivated) to perform the task because the stimuli were not intense or appealing enough to attract their attention. However, as mentioned in the introduction, successful performance on a sustained attention task requires that one is able to endogenously modulate alertness by resisting to potentially interfering effects or distracting stimuli $(13,14)$ in such situations. Therefore, a complementary interpretation holds that, during the processing of stimuli with repetitive, nonarousing 
qualities, boys in the high-anxiety group were less able to resist to such emerging internal and external distractions, resulting in more off-task behavior (e.g. thinking about other things, looking away) and more variable reaction times. These results fit previous results of our study (12) where it was shown that adolescents of highly anxious mothers were unable to endogenously generate response control processes in a divided-attention task. This kind of endogenous control is also needed to perform successfully in a CPT. Thus, it seems that the ADHD deficit triggered by antenatal maternal anxiety only appears in tasks that challenge the executive processes by which behavior must be internally regulated (26). The distinction between endogenous and exogenous response control processes receives support from the fact that several functions involving the PFC have been shown to involve different areas depending upon whether they were externally or internally generated $(12,27-29)$.

The observed sustained attention difficulties could possibly be ascribed to medial PFC (including ACC). However, the deficit could also be related to a dysfunction of subcortical systems interacting with PFC such as the basal ganglia, thalamus, limbic system, and brainstem, as indicated by blood flow and electroencephalographic studies (30).

We found the period between 12 and 22 wk postmenstrual age to be critical in revealing an association between maternal anxiety and sustained attention/self-regulation in boys. In humans, important brain developmental processes such as neuron proliferation, migration and differentiation take place between 8 and 24 wk postmenstrual age in brain areas connected to the PFC (e.g. hippocampus, amygdala, ACC, brainstem, basal ganglia) (31-33). Therefore, it is plausible to state that later PFC functioning may be altered by physiologic correlates of the mother's emotional state when the latter brain circuits were developing. However, we do not know what the level of antenatal maternal anxiety was before $12 \mathrm{wk}$ of gestation.

Strengths and limitations. A strength of the current results is that they corroborate results concerning self-regulation problems (9), impulsivity, and deficits in endogenous inhibition (12). High maternal anxiety between 12 and 22 wk was related to self-regulation problems as expressed in ADHD symptoms (inattention, impulsivity, disinhibition), externalizing problems (conduct disorders, aggression), and internalizing problems (emotional inhibition and lability, high subjective distress in novel situations) in 8/9 y olds. Our results particularly corroborate the results on ADHD symptoms in the 8/9 and 14/15 y olds for the following reasons: 1) A drop in performance efficiency over time (related to inappropriately timed and poor response control) on CPT is a hallmark of ADHD (34). 2) Behavioral genetic studies consider variable responding on CPT to be the phenotype of ADHD (35). 3) The present finding that boys and not girls of highly anxious pregnant mothers performed worse on the CPT bolsters the clinical observations that many more boys than girls have ADHD (36). Thus, based on the present results, it is tempting to suggest again that, under certain conditions, high antenatal maternal anxiety plays a role in the etiology of ADHD. Most probably the interaction between the genetic susceptibility of the mother and/or her offspring and the physiologic variables associated with antenatal maternal anxiety are crucial (9).

Caution is needed with respect to the results for the girls because there were only 5 girls in the highly anxious group compared with 11 boys, resulting in high error variances and making it difficult to observe/interpret any effects. The results regarding the boys are to be considered particularly strong for two reasons. First, there was no difference in maternal anxiety level between boys and girls in the high-anxiety group. Second, just like boys of highly anxious mothers, girls of highly anxious mothers were shown to be susceptible to the negative influence of their mother's emotional state during pregnancy in all waves of the study up to now. In addition, it has to be noted that the total high-anxiety group, comprising only 16 subjects, is rather small.

In conclusion, the results of this study corroborate the evidence provided by a growing number of studies that maternal emotional states during pregnancy can influence cognitive, behavioral, and emotional development of the fetus, child, and even the adolescent.

Acknowledgments. The authors thank the mothers, fathers, children, and teachers who took part in the study.

\section{REFERENCES}

1. Huizink AC, Mulder EJ, Buitelaar JK 2004 Prenatal stress and risk for psychopathology: specific effects or induction of general susceptibility? Psychol Bull 130:115-142

2. Van den Bergh BR, Mulder EJ, Mennes M, Glover V 2005 Antenatal maternal anxiety and stress and the neurobehavioural development of the fetus and child: links and possible mechanisms. A review. Neurosci Biobehav Rev 29:237-258

3. Weinstock M 2005 The potential influence of maternal stress hormones on development and mental health of the offspring. Brain Behav Immun 19:296-308

4. Brouwers EPM, van Baar AL, Pop VJM 2001 Maternal anxiety during pregnancy and subsequent infant development. Infant Behavior Dev 24:95-106

5. Huizink AC, de Medina PR, Mulder EJ, Visser GH, Buitelaar JK 2002 Prenatal maternal stress, HPA-axis activity, and postnatal infant development. In: Sivik T, Byrne D, Lipsitt D, Christodoulou G, Dienstfrey H (eds) Psycho-Neuro-EndocrinoImmunology (PNEI). Medica International Congress Series. Elsevier, Amsterdam, 1241:65-71

6. Huizink AC, Robles de Medina PG, Mulder EJ, Visser GH, Buitelaar JK 2003 Stress during pregnancy is associated with developmental outcome in infancy. J Child Psychol Psychiatry 44:810-818

7. O'Connor TG, Heron J, Golding J, Beveridge M, Glover V 2002 Maternal antenatal anxiety and children's behavioural/emotional problems at 4 years: Report from the Avon Longitudinal Study of Parents and Children. Br J Psychiatry 180:502-508

8. O'Connor TG, Heron J, Golding J, Glover V, The ALSPAC Study Team 2003 Maternal anxiety and behavioural/emotional problems in children: a test of a programming hypothesis. J Child Psychol Psychiatry 44:1025-1036

9. Van den Bergh BR, Marcoen A 2004 High antenatal maternal anxiety is related to ADHD symptoms, externalizing problems, and anxiety in 8- and 9-year-olds. Child Dev 75:1085-1097

10. Seckl JR 2001 Glucocorticoid programming of the fetus; adult phenotypes and molecular mechanisms. Mol Cell Endocrinol 185:61-71

11. Seckl JR 2004 Prenatal glucocorticoids and long-term programming. Eur J Endocrinol 151:U49-U62

12. Van den Bergh BR, Mennes M, Oosterlaan J, Stevens V, Stiers P, Marcoen A, Lagae L 2005 High antenatal maternal anxiety is related to impulsivity during performance on cognitive tasks in 14- and 15-year-olds. Neurosci Biobehav Rev 29:259-269

13. Robertson IH, Manly T, Andrade J, Baddeley BT, Yiend J 1997 'Oops!': performance correlates of everyday attentional failures in traumatic brain injured and normal subjects. Neuropsychologia 35:747-758

14. Fernandez-Duque D, Posner MI 2001 Brain imaging and attentional networks in normal and pathological states. J Clin Exp Neuropsychol 23:74-93

15. Paus T 2001 Primate anterior cingulate cortex: where motor control, drive and cognition interface. Nat Rev Neurosci 2:417-424

16. Posner MI, Rothbart MK 1998 Attention, self-regulation, and consciousness. Philos Trans R Soc Lond B Biol Sci 353:1915-1927

17. Eisenberg N, Spinrad TL 2004 Emotion-related regulation: sharpening the definition. Child Dev 75:334-339 
18. Spielberger CD, Gorsuch RL, Lushene RE 1970 STAI Manual for the State Trait Anxiety Inventory. Consulting Psychologists Press, Palo Alto, CA

19. Van der Ploeg HM, Defares PB, Spielberger CD 1980 Manual of the Self-Report Questionnaire. Swets \& Zeitlinger B.V., Lisse, The Netherlands

20. Spielberger C 1975 The measurement of state and trait Anxiety: conceptual and methodological issues. In: Levi L (ed) Emotions: Their Parameters and Measurement. Raven, New York, pp 712-725.

21. Van der Meere J, Shalev R, Borger N, Gross-Tsur V 1995 Sustained attention, activation and MPH in ADHD: a research note. J Child Psychol Psychiatry 36:697-703

22. Borger N, Van der Meere J 2000 Visual behaviour of ADHD children during an -attention test: an almost forgotten variable. Attention-deficit hyperactivity disorder. J Child Psychol Psychiatry 41:525-532

23. van Haasen PP, de Bruyn EE, Pijl YL, Poortinga YH, lutje Spelberg HC, Vander Steene G, Coetsier P, Spoelders-Claes R, Stinissen J 1986 Wechsler Intelligence Scale for Children-Revised. Nederlandstalige uitgave. Swets \& Zeitlinger, Lisse, The Netherlands

24. Wechsler D 1974 Manual for the Wechsler Intelligence Scale for Children-Revised. The Psychological Corporation, New York

25. Grossman AW, Churchill JD, McKinney BC, Kodish IM, Otte SL, Greenough WT 2003 Experience effects on brain development: possible contributions to psychopathology. J Child Psychol Psychiatry 44:33-63

26. Nigg JT 2001 Is ADHD a disinhibitory disorder? Psychol Bull 127:571-598

27. Fuster JM 2001 The prefrontal cortex-an update: time is of the essence. Neuron 30:319-333
28. Uylings HB, Groenewegen HJ, Kolb B 2003 Do rats have a prefrontal cortex? Behav Brain Res 146:3-17

29. Miller EK, Cohen JD 2001 An integrative theory of prefrontal cortex function. Annu Rev Neurosci 24:167-202

30. Riccio CA, Reynolds CR, Lowe P, Moore JJ 2002 The continuous performance test: a window on the neural substrates for attention? Arch Clin Neuropsychol 17:235272

31. Levitt $P 2003$ Structural and functional maturation of the developing primate brain J Pediatr 143:35-45

32. Nowakowski RS, Hayes NL 2002 General principles of CNS development. In: Johnson MH, Munakata Y, Gilmore ROE (eds) Brain Development and Cognition. A Reader. Blackwell Publishers, Malden pp 57-82

33. Rakic P 2002 Pre- and post-developmental neurogenesis in primates. Clin Neurosci Res 2:29-39

34. Rubia K 2002 The dynamic approach to neurodevelopmental psychiatric disorders: use of fMRI combined with neuropsychology to elucidate the dynamics of psychiatric disorders, exemplified in ADHD and schizophrenia. Behav Brain Res 130:47-56

35. Kunsti J, Stevenson J 2001 Psychological mechanisms in hyperactivity: II. The role of genetic factors. J Child Psychol Psychiatry 42:211-219

36. Anderson JC, Williams S, McGee R, Silva PA 1987 DSM-III disorders in preadolescent children. Prevalence in a large sample from the general population. Arch Gen Psychiatry 44:69-76 